\title{
Modelo de monitoreo de comportamientos inusuales o conglomerados de defectos congénitos en el nivel departamental, distrital y municipal, Colombia, 2018
}

\section{Monitoring model of unusual behaviors or conglomerates of birth defects at the departmental, district and municipal levels, Colombia, 2018}

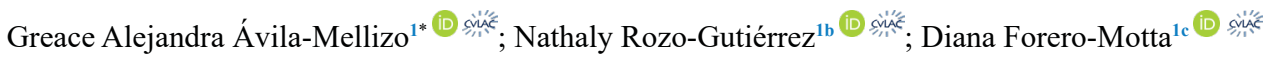 \\ *gavilam@ins.gov.co
}

Forma de citar: Ávila Mellizo GA, Rozo Gutiérrez N, Forero Motta D. Modelo de monitoreo de comportamientos inusuales o conglomerados de defectos congénitos en el nivel departamental, distrital y municipal, Colombia, 2018. Salud UIS. 2022; e22015. doi: https://doi.org/10.18273/saluduis.54.e:22015 (c) (i)

\section{Resumen}

Introducción: la información de la vigilancia en salud pública permite la detección de patrones inusuales en los datos, con el fin de que las respuestas de salud pública sean oportunas y contribuyan a la disminución de la morbimortalidad de la población infantil. Objetivos: describir un modelo de monitoreo diseñado para la identificación de comportamientos inusuales y conglomerados de defectos congénitos, así como el incremento en la frecuencia del evento en comparación con el número de casos notificados históricamente. Materiales y Métodos: estudio descriptivo retrospectivo que incluye el análisis de los casos notificados al Sistema Nacional de Vigilancia en Salud Pública (SIVIGILA) durante los últimos tres años, agrupados y comparados con las prevalencias de acuerdo con lo reportado por el ECLAM. Se usó la distribución de probabilidades de Poisson y se identificó aquellas entidades territoriales en donde se observan diferencias significativas entre lo esperado y lo observado con un valor de significancia $<0,05(\mathrm{p}<0,05)$. Resultados: se identificaron prevalencias superiores en holoprosencefalia, microcefalia, polidactilia, defectos por reducción de miembro inferior y coartación de la aorta. Los comportamientos inusuales de los defectos congénitos de sistema nervioso central se concentran en anencefalia, espina bífida, hidrocefalia, microcefalia. Conclusiones: las condiciones particulares de cada municipio y/o departamento y el comportamiento de los defectos congénitos en algunas áreas podría indicar que las mujeres en estado de gestación son susceptibles a mayores riesgos en zonas particulares, y que este riesgo en particular podría ser el resultado de diversas inequidades en salud generadas por las interacciones sociales, ambientales y comportamentales.

Palabras clave: Vigilancia en Salud Pública; Anomalías congénitas; Salud pública; Mortalidad infantil; Mortalidad perinatal; Mortalidad prematura; Discapacidades del desarrollo.

\footnotetext{
${ }^{1}$ Instituto Nacional de Salud. Bogotá, Colombia
} 


\begin{abstract}
Introduction: The public health surveillance information allows the detection of unusual patterns in the data in order that public health responses are timely and contribute to the reduction of morbidity and mortality of the child population. Objectives: To describe a monitoring model designed for the identification of unusual behaviors and conglomerates of congenital defects, as well as the increase in the frequency of the event in comparison with the number of cases reported historically. Materials and Methods: A retrospective descriptive study that includes the analysis of the cases notified to the Public Health National Surveillance System (SIVIGILA) during the last three years, grouped and compared with the prevalences according to what was reported by the ECLAM. The distribution of Poisson probabilities was used identifying those territorial entities where significant differences were observed between what was expected and what was observed with a value of significance $<0.05(p<0.05)$. Results: Superior prevalences were identified in holoprosencephaly, microcephaly, polydactyly, defects due to reduction of the lower limb and coarctation of the aorta. Unusual behaviors of congenital defects of the central nervous system are concentrated in anencephaly, spina bifida, hydrocephalus, microcephaly. Conclusions: The conditions of each municipality and / or department and the behavior of congenital defects in some areas could indicate that women in pregnancy are susceptible to greater risks areas, and that this risk could be the result of various health inequities generated by social, environmental, and behavioral interactions.
\end{abstract}

Keywords: Public health surveillance; Congenital anomalies; Public health; Infant mortality; Perinatal mortality; Mortality-premature; Developmental disabilities.

\section{Introducción}

En las últimas dos décadas las malformaciones congénitas pasan de ser la sexta a la segunda causa de muerte en menores de cinco años. Es uno de los eventos en salud pública que mayor pérdida de años de vida por muerte prematura y discapacidad genera en el mundo, además del impacto social, económico y emocional que producen en los afectados, sus familias, los sistemas de salud y la sociedad ${ }^{1}$.

Se ha descrito en los últimos años que a nivel global las malformaciones congénitas tienen una frecuencia aproximada del $3 \%$ en recién nacido vivos y entre el $10 \%$ y el $15 \%$ en mortinatos ${ }^{2,3}$. En las Américas las anomalías congénitas son la segunda causa de muerte en los niños menores de 5 años ${ }^{4}$. En Colombia, según las estadísticas vitales del Departamento Administrativo Nacional de Estadística (DANE), en el 2006, las malformaciones congénitas fueron la segunda causa de mortalidad en menores de un año; provocaron el 20,8\% de las muertes ${ }^{5}$. Así mismo, la prevalencia de los defectos congénitos en Colombia para 2017 fue de 113,6 casos por cada 10000 nacidos vivos. Por grupos de malformaciones congénitas la prevalencia más alta se concentra en el sistema osteomuscular (27,9 casos), sistema circulatorio $(23,2$ casos $)$ y el sistema nervioso $(16,3 \text { casos })^{6}$.

Bajo este contexto, desde el 2010 la Organización Mundial de la Salud (OMS) en la $63^{\circ}$ Asamblea Mundial de la Salud establece la necesidad del desarrollo de sistemas de vigilancia en salud pública de defectos congénitos, con el propósito de que los países dispongan de información confiable para reconocer su prevalencia entre las comunidades como marco orientador para la toma de decisiones en salud pública $^{7}$. Lo anterior se ha desarrollado por medio de sistemas de vigilancia que permiten la recopilación, el análisis, la interpretación y la difusión continúa y sistemática de datos, así como también el monitoreo continuo del comportamiento epidemiológico, en este caso de los defectos congénitos, para la identificación de las tasas de prevalencia e incidencia, que fluctúan en las poblaciones a lo largo del tiempo, lo que facilita la identificación de conglomerados, los cuales corresponden a una agregación real o percibida de más de la cantidad esperada de casos de defectos congénitos en una población durante un período específico. De esta forma la evidencia derivada del proceso de vigilancia justifica la generación de alertas por enfermedades emergentes o re-emergentes, el control del riesgo poblacional para orientar acciones en salud pública tendientes a la reducción de la morbimortalidad y la discapacidad evitable ${ }^{8-10}$.

En este documento se describe un modelo de monitoreo diseñado para la identificación de comportamientos inusuales o conglomerados de defectos congénitos, y el incremento en la frecuencia del evento en comparación con el número de casos notificados históricamente para un territorio en un periodo determinado de tiempo. 


\section{Materiales y métodos}

\section{Estudio descriptivo retrospectivo.}

La información evaluada se obtiene por medio de la notificación individual semanal de casos al Sistema de Vigilancia en Salud Pública (SIVIGILA) del evento 215 - defectos congénitos, realizada por las unidades primarias generadoras de datos (UPGD) a nivel nacional, las cuales identifican y configuran el caso, de acuerdo con la definición de caso para el evento.

Para el análisis se incluyó la información correspondiente a la notificación de casos entre 2015 y hasta el periodo epidemiológico 6 de 2018. La información notificada se sometió a un proceso de depuración, verificación de diagnósticos, completitud, consistencia y veracidad. Se realizó la validación y filtrado de los datos y se eliminaron aquellos casos notificados repetidos. Los casos repetidos se identifican con formato condicional de valores duplicados en la variable número de identificación del menor notificado. Posteriormente, se revisa la variable: embarazo múltiple, que permite identificar los casos de embarazos múltiples y casos gemelares, para estos casos se mantienen los dos registros. Consecutivamente, se verifican los tipos de defectos congénitos en los casos repetidos, ya que si hay un registro ingresado con un defecto metabólico y otro con una malformación se deben dejar los dos registros. Para aquellos que corresponden a repetidos, se revisan los casos y se deja solo uno, teniendo en cuenta los siguientes criterios: a) si hay un caso con clasificación 6 o D (descartados), se deja el otro registro; b) si los casos tienen la misma clasificación, se revisa la condición final, y el que tenga en condición final "muerto" se deja como caso; c) si tienen la misma condición final, queda el caso que este confirmado con ajuste "3 (confirmado por laboratorio)" o "4 (confirmado por clínica)" en "ajuste" o tipo de caso "tip_cas"; d) si tienen la misma clasificación en tipo de caso y el mismo ajuste, se verifica la calidad de la información y se deja el que tenga la mejor definición del diagnóstico y código CIE 10. Finalmente, si los criterios anteriores no permiten definir qué caso dejar, se escoge el que tenga la fecha de notificación más reciente.

El análisis geográfico de los casos se realizó de acuerdo con la entidad territorial (departamento, distrito y municipio) de residencia.

Los defectos congénitos fueron clasificados en seis grandes grupos basados en la clasificación de las malformaciones congénitas del Estudio Colaborativo
Latino Americano de Malformaciones Congénitas (ECLAMC) que permite tener grupos diagnósticos comparables $^{11}$ (Tabla 1). El estimado de la prevalencia por cada uno de los grupos de defectos congénitos notificados al sistema de vigilancia de Colombia fue comparado con las prevalencias calculadas por el ECLAMC para América del Sur.

Para la identificación de comportamientos inusuales en cada grupo de defectos se usó la distribución de probabilidades de Poisson, dado que corresponde a un evento de baja prevalencia. Se estableció un valor esperado para cada defecto congénito priorizado, el cual correspondió al valor medio de casos de las 52 semanas epidemiológicas de los años 2015, 2016 y 2017, y un valor observado, que correspondió al número de casos de la semana en estudio (semana epidemiológica 24) más las 51 semanas epidemiológicas anteriores, lo que completa el periodo de análisis de 52 semanas. Por medio de la estimación de la probabilidad de ocurrencia del evento según su comportamiento medio de los últimos tres años, se logró identificar aquellos municipios con diferencias significativas entre lo esperado y lo observado con un valor de significancia $<0,05(\mathrm{p}<0,05)$.

En este escenario se consideró un conglomerado municipal de defectos congénitos en los siguientes escenarios:

- Municipios con un número de casos observados superior al número de casos esperados y se obtenga un valor de Poisson menor de 0,05 .

- Municipios con un número de casos esperados (media) igual a cero y un número de casos observados mayor o igual a dos.

Para identificación y visualización de nuevos patrones y relaciones geográficas de conglomerado de defectos o grupos de defectos congénitos priorizados, se usan las relaciones espaciales de vecindad, bajo el criterio de contigüidad. De esta forma se considera un conglomerado espacial en aquellos casos en donde se involucren dos o más municipios contiguos con comportamiento inusuales.

Los datos obtenidos fueron procesados en el software Epi-Info versión 7.2. Los resultados se organizaron en distribución de frecuencia, prevalencia y se presentan en tablas, figuras y mapas. 
Tabla 1. Priorización de los defectos congénitos en seguimiento, Colombia, 2018.

\begin{tabular}{|c|c|c|}
\hline Grupo (a) & Defecto congénito (b) & Código CIE 10 \\
\hline \multirow{6}{*}{ Sistema Nervioso Central } & Anencefalia & $\mathrm{Q} 000$ \\
\hline & Encefalocele & Q010 - Q011 - Q012 - Q018 - Q019 \\
\hline & Espina bífida & Q050 a Q059 \\
\hline & Hidrocefalia & Q030 - Q031 - Q038 - Q039 \\
\hline & Holoprosencefalia & Q042 \\
\hline & Microcefalia & Q02 \\
\hline \multirow{2}{*}{ Sistema osteomuscular } & Gastrosquisis & Q793 \\
\hline & Onfalocele & Q792 \\
\hline \multirow{7}{*}{ Defectos extremidades } & Deformidad congénita de los pies & Q660 A Q669 \\
\hline & Polidactilia & Q690 - Q691 - Q692 - Q699 \\
\hline & Sindactilia & $\begin{array}{c}\text { Q700 - Q701 - Q702 - Q703 - Q704 - } \\
\text { Q709 }\end{array}$ \\
\hline & $\begin{array}{c}\text { Defectos por reducción del miembro } \\
\text { superior }\end{array}$ & Q710 A Q719 \\
\hline & $\begin{array}{c}\text { Defectos por reducción del miembro } \\
\text { inferior }\end{array}$ & Q720 A Q729 \\
\hline & $\begin{array}{l}\text { Osteocondrodisplasia con defecto del } \\
\text { crecimiento de los huesos largos y de la } \\
\text { columna vertebral }\end{array}$ & Q770 A Q779 \\
\hline & Osteogenesis imperfecta & Q780 \\
\hline \multirow{3}{*}{ Sistema circulatorio } & Coartación de la aorta & Q251 \\
\hline & Tetralogía de Fallot & Q213 \\
\hline & $\begin{array}{l}\text { Transposición de los grandes vasos del } \\
\text { ventrículo derecho }\end{array}$ & Q201 - Q202 \\
\hline \multirow{3}{*}{ Fisura de labio y paladar } & Fisura de paladar & Q351 - Q353 - Q355 - Q357 - Q359 \\
\hline & Labio leporino & Q360 - Q361 - Q369 \\
\hline & Fisura del paladar con labio leporino & Q370 A Q375 -Q378 - Q379 \\
\hline \multirow{3}{*}{ Defectos cromosómicos } & Síndrome Down & Q900 - Q901 - Q902 - Q909 \\
\hline & Síndrome de Edwards & Q910 A Q913 \\
\hline & Síndrome Patau & Q914 A Q917 \\
\hline
\end{tabular}

Fuente: SIVIGILA, Instituto Nacional de Salud, Colombia

\section{Resultados}

\section{Análisis comparativo de prevalencias por defecto congénito}

El estimado de la prevalencia para cada grupo de defectos congénitos fue comparado con las prevalencias calculadas por el ECLAMC para América del Sur. Para Colombia, se identificaron prevalencias superiores en holoprosencefalia, microcefalia, polidactilia, defectos por reducción de miembro inferior y coartación de la aorta, esta última con diferencias en los cuatro periodos en estudio (Tabla 2).

\section{Identificación de comportamientos inusuales}

Se realizó la identificación de comportamientos inusuales por cada grupo de defectos congénitos. En el grupo de defectos congénitos del sistema nervioso central se identificaron comportamientos inusuales en 17 municipios con un valor esperado de cero y un valor observado de dos o más, y en seis municipios con un valor observado superior al esperado y un valor de $p$ menor a 0,05 . Para el caso de anencefalia se identificó comportamiento inusual en cinco municipios, para espina bífida en tres municipios, hidrocefalia en ocho municipios y microcefalia en siete municipios. No se identificaron comportamientos inusuales para notificación de encefalocele y holoprosencefalia (Tabla 3).

Para el grupo de defectos congénitos de extremidades, se identificó comportamiento inusual en 26 municipios de 16 departamentos. En las deformidades congénitas de los pies se identificó comportamiento inusual en 14 municipios, en polidactilia en siete municipios, en sindactilia en cuatro y en los defectos por reducción del miembro superior y osteocondrodisplasia se identificó comportamiento inusual en dos municipios cada uno. 
No se identificó comportamiento inusual en los defectos por reducción del miembro inferior ni en osteogénesis imperfecta (Tabla 4).

En los defectos congénitos del sistema osteomuscular se identificaron comportamientos inusuales de notificación de gastrosquisis en Arauca y Nariño y de onfalocele en Acacias - Meta. Para los dos defectos congénitos se tienen valores esperados de cero y observados de dos y tres casos (Tabla 5).

Para los defectos congénitos del sistema circulatorio se identificaron comportamientos inusuales en cinco municipios de cuatro departamentos. En el caso de coartación de la aorta los comportamientos inusuales se identificaron en los departamentos de Antioquia, Boyacá y Santander, mientras que para la trasposición de grandes vasos se identificó un comportamiento inusual en el municipio de Pereira - Risaralda. No se identificaron comportamientos inusuales para tetralogía de Fallot (Tabla 6).

En el grupo de defectos congénitos relacionados con fisura palatina y fisura del labio se identificaron comportamientos inusuales en 17 municipios en 14 departamentos. El departamento con el mayor número de municipios con comportamientos inusuales fue Antioquia, en donde se identificaron Caldas e Itagüí para fisura de paladar, y Dabeiba y Granada para fisura paladar y labio leporino (Tabla 7).

En el grupo de defectos congénitos de etiología cromosómica no se identificaron comportamientos inusuales en la notificación de casos de síndrome Edwards ni síndrome de Patau. Para el caso de síndrome de Down se identificaron comportamientos inusuales en 21 municipios de 14 departamentos (Tabla 8).

Tabla 2. Prevalencia (por 10000 nacidos vivos) de defectos congénitos priorizados, Colombia, 2015 a primer semestre 2018.

\begin{tabular}{|c|c|c|c|c|c|c|}
\hline \multirow[b]{2}{*}{ Grupo } & \multirow[b]{2}{*}{ Defecto congénito } & \multirow[b]{2}{*}{$\begin{array}{l}\text { Prevalencia } \\
\text { ECLAMC }\end{array}$} & \multicolumn{4}{|c|}{ Prevalencia Colombia } \\
\hline & & & 2015 & 2016 & 2017 & $\begin{array}{c}2018 \\
\text { I Sem. }\end{array}$ \\
\hline \multirow{6}{*}{$\begin{array}{l}\text { Sistema nervioso } \\
\text { central }\end{array}$} & Anencefalia & 5,79 & 3,15 & 1,99 & 1,87 & 1,88 \\
\hline & Encefalocele & 3,28 & 0,82 & 0,97 & 0,59 & 0,50 \\
\hline & Espina bífida & 8,46 & 1,42 & 1,54 & 1,41 & 1,28 \\
\hline & Hidrocefalia & 17,21 & 3,30 & 4,42 & 3,89 & 3,09 \\
\hline & Holoprosencefalia & 0,78 & 0,67 & 0,78 & 0,93 & 0,66 \\
\hline & Microcefalia & 4,83 & 1,66 & 8,96 & 5,95 & 3,85 \\
\hline \multirow{2}{*}{ Sistema osteomuscular } & Gastrosquisis & 9,59 & 1,92 & 2,55 & 3,40 & 2,81 \\
\hline & Onfalocele & 4,54 & 0,80 & 0,90 & 0,90 & 1,06 \\
\hline \multirow{7}{*}{ Defectos extremidades } & Deformidad congénita de los pies & 7,60 & 5,49 & 6,30 & 6,61 & 5,56 \\
\hline & Polidactilia & 3,14 & 5,66 & 7,00 & 7,54 & 3,63 \\
\hline & Sindactilia & Sin dato & 1,77 & 1,84 & 1,65 & 1,88 \\
\hline & $\begin{array}{l}\text { Defectos por reducción del } \\
\text { miembro superior }\end{array}$ & 1,10 & 0,92 & 1,14 & 1,00 & 0,97 \\
\hline & $\begin{array}{l}\text { Defectos por reducción del } \\
\text { miembro inferior }\end{array}$ & 0,35 & 1,29 & 0,54 & 0,96 & 0,19 \\
\hline & Osteocondrodisplasia & Sin dato & 0,70 & 0,97 & 0,85 & 0,84 \\
\hline & Osteogenesis imperfecta & Sin dato & 0,27 & 0,19 & 0,25 & 0,13 \\
\hline \multirow{3}{*}{ Sistema circulatorio } & Coartación de la aorta & 0,52 & 0,98 & 1,16 & 1,51 & 1,22 \\
\hline & Tetralogía de Fallot & 1,64 & 0,71 & 0,91 & 1,11 & 0,94 \\
\hline & Transposición de los grandes vasos & 0,66 & 0,05 & 0,26 & 0,19 & 0,22 \\
\hline \multirow{3}{*}{$\begin{array}{l}\text { Fisura de labio y } \\
\text { paladar }\end{array}$} & Fisura de paladar & 4,46 & 1,48 & 1,62 & 1,91 & 1,50 \\
\hline & Labio leporino & 11,72 & 2,62 & 2,81 & 2,93 & 2,06 \\
\hline & $\begin{array}{c}\text { Fisura del paladar con labio } \\
\text { leporino }\end{array}$ & 11,72 & 3,10 & 3,44 & 3,64 & 3,28 \\
\hline \multirow{3}{*}{$\begin{array}{l}\text { Defectos } \\
\text { cromosómicos }\end{array}$} & Síndrome de Down & 17,70 & 6,22 & 6,90 & 7,86 & 6,03 \\
\hline & Síndrome de Edwards & 1,32 & 0,51 & 0,22 & 0,42 & 0,34 \\
\hline & Síndrome de Patau & 0,57 & 0,17 & 0,25 & 0,20 & 0,25 \\
\hline
\end{tabular}

Fuente: SIVIGILA - INS, 2015-2018. DANE. ECLAMC 
Tabla 3. Entidades territoriales con comportamientos inusuales en el grupo de defectos congénitos del sistema nervioso central, Colombia, 2015 - I semestre 2018

\begin{tabular}{|c|c|c|c|c|}
\hline Departamento & Municipio & Media & Observado & Poisson \\
\hline \multicolumn{5}{|l|}{ Anencefalia } \\
\hline Antioquia & Turbo & 0 & 2 & 0,04 \\
\hline Arauca & Arauca & 0 & 2 & 0,04 \\
\hline Cauca & Popayán & 1 & 3 & 0,03 \\
\hline \multirow[t]{2}{*}{ Norte de Santander } & Ocaña & 0 & 2 & 0,00 \\
\hline & Villa del Rosario & 0 & 2 & 0,00 \\
\hline \multicolumn{5}{|l|}{ Espina bífida } \\
\hline Antioquia & Cáceres & 0 & 2 & 0,00 \\
\hline Arauca & Arauca & 0 & 2 & 0,00 \\
\hline Cesar & Tamalameque & 0 & 2 & 0,04 \\
\hline \multicolumn{5}{|l|}{ Hidrocefalia } \\
\hline Amazonas & Leticia & 1 & 3 & 0,03 \\
\hline Antioquia & Antioquia & 1 & 8 & 0,00 \\
\hline Bolivar & Villanueva & 0 & 2 & 0,04 \\
\hline Cauca & Sucre & 0 & 3 & 0,00 \\
\hline Cundinamarca & Silvania & 0 & 2 & 0,04 \\
\hline Santander & Villanueva & 0 & 2 & 0,04 \\
\hline \multirow[t]{2}{*}{ Sucre } & San Benito & 0 & 2 & 0,04 \\
\hline & Sucre & 0 & 3 & 0,00 \\
\hline \multicolumn{5}{|l|}{ Microcefalia } \\
\hline Antioquia & Apartado & 0 & 2 & 0,04 \\
\hline Casanare & Tauramena & 1 & 5 & 0,01 \\
\hline Huila & Pital & 0 & 2 & 0,04 \\
\hline Nariño & Córdoba & 0 & 3 & 0,00 \\
\hline \multirow[t]{2}{*}{ Sucre } & Sincelejo & 5 & 10 & 0,01 \\
\hline & Sucre & 1 & 8 & 0,00 \\
\hline Valle del Cauca & Pradera & 0 & 2 & 0,00 \\
\hline
\end{tabular}

Fuente: SIVIGILA - INS, 2015-2018.

Tabla 4. Entidades territoriales con comportamientos inusuales en el grupo de defectos congénitos de extremidades, Colombia, 2015 - I semestre 2018

\begin{tabular}{ccccc}
\hline Departamento & Municipio & Media & Observado & Poisson \\
\hline Deformidad congénita de los pies & & & 0,04 \\
\hline Antioquia & Apartado & 0 & 2 & 0,00 \\
& Envigado & 0 & 3 & 0,04 \\
Atlántico & Malambo & 0 & 2 & 0,04 \\
Bolivar & Santa Rosa del Sur & 0 & 2 & 0,00 \\
Cauca & Bolivar & 0 & 3 & 0,00 \\
& Padilla & 0 & 2 & 0,02 \\
Guainía & Puerto Inírida & 1 & 4 & 0,04 \\
Nariño & Chachagui & 0 & 2 & 0,00 \\
Putumayo & Córdoba & 0 & 4 & 0,00 \\
Santander & Puerto Leguizamo & 1 & 5 & 0,00 \\
Sucre & Floridablanca & 1 & 4 & 0,00 \\
& Girón & 0 & 3 & 0,04 \\
\end{tabular}


Modelo de monitoreo de comportamientos inusuales o conglomerados de defectos congénitos en el nivel departamental, distrital y municipal, Colombia, 2018

\begin{tabular}{|c|c|c|c|c|}
\hline Departamento & Municipio & Media & Observado & Poisson \\
\hline \multicolumn{5}{|l|}{ Polidactilia } \\
\hline Antioquia & Caldas & 0 & 9 & 0,00 \\
\hline Arauca & Arauca & 0 & 2 & 0,04 \\
\hline Bolivar & San Pablo & 0 & 2 & 0,04 \\
\hline Cauca & Bolivar & 1 & 18 & 0,00 \\
\hline Huila & Palestina & 0 & 2 & 0,00 \\
\hline Santander & Floridablanca & 0 & 2 & 0,00 \\
\hline Valle del Cauca & Cali & 20 & 25 & 0,04 \\
\hline \multicolumn{5}{|l|}{ Sindactilia } \\
\hline Antioquia & Medellín & 3 & 6 & 0,03 \\
\hline Arauca & Arauca & 0 & 2 & 0,04 \\
\hline Boyaca & Tunja & 1 & 5 & 0,01 \\
\hline Risaralda & Santa Rosa de Cabal & 0 & 2 & 0,04 \\
\hline \multicolumn{5}{|c|}{ Defecto por reducción de miembro superior } \\
\hline Cundinamarca & Funza & 0 & 2 & 0,00 \\
\hline Santander & Bucaramanga & 0 & 2 & 0,04 \\
\hline \multicolumn{5}{|c|}{ Osteocondrodisplasia } \\
\hline Cesar & Valledupar & 0 & 2 & 0,04 \\
\hline Sucre & Sincelejo & 0 & 3 & 0,00 \\
\hline
\end{tabular}

Fuente: SIVIGILA - INS, 2015-2018.

Tabla 5. Entidades territoriales con comportamientos inusuales en el grupo de defectos congénitos del sistema osteomuscular, Colombia, 2015 - I semestre 2018

\begin{tabular}{ccccc}
\hline Departamento & Municipio & Media & Observado & Poisson \\
\hline Gastrosquisis & & & & \\
\hline Arauca & Arauca & 0 & 3 & 0,04 \\
Nariño & Nariño & 0 & & 0,00 \\
\hline Onfalocele & & & 2 & 0,00 \\
\hline Meta & Acacias & 0 & & \\
\hline
\end{tabular}

Fuente: SIVIGILA - INS, 2015-2018.

Tabla 6. Entidades territoriales con comportamientos inusuales en el grupo de defectos congénitos del sistema circulatorio, Colombia, 2015 - I semestre 2018

\begin{tabular}{ccccc}
\hline Departamento & Municipio & Media & Observado & Poisson \\
\hline Coartación de la aorta & & & 2 & 0,04 \\
\hline Antioquia & Envigado & 0 & 5 & 0,00 \\
Boyacá & Boyacá & 1 & 5 & 0,01 \\
Santander & Bucaramanga & 1 & 2 & 0,04 \\
& Floridablanca & 0 & & \\
\hline Trasposición de grandes vasos & & 2 & 0,04 \\
\hline \multicolumn{2}{r}{ Risaralda } & Pereira & 0 & \\
\hline
\end{tabular}

Fuente: SIVIGILA - INS, 2015-2018. 
Tabla 7. Entidades territoriales con comportamientos inusuales en el grupo de defectos congénitos de fisura de labio y paladar, Colombia, 2015 - I semestre 2018

\begin{tabular}{|c|c|c|c|c|}
\hline Departamento & Municipio & Media & Observado & Poisson \\
\hline \multicolumn{5}{|l|}{ Fisura de paladar } \\
\hline \multirow[t]{2}{*}{ Antioquia } & Caldas & 0 & 5 & 0,00 \\
\hline & Itagüí & 0 & 2 & 0,04 \\
\hline Bolivar & Calamar & 0 & 2 & 0,04 \\
\hline Huila & Pitalito & 1 & 5 & 0,00 \\
\hline Sucre & Sincelejo & 0 & 2 & 0,04 \\
\hline \multicolumn{5}{|l|}{ Labio leporino } \\
\hline Cundinamarca & La Mesa & 0 & 2 & 0,04 \\
\hline La Guajira & Manaure & 0 & 2 & 0,00 \\
\hline Nariño & Córdoba & 0 & 6 & 0,00 \\
\hline Tolima & Ibagué & 2 & 6 & 0,02 \\
\hline Valle del Cauca & Bolivar & 0 & 5 & 0,00 \\
\hline Vichada & Cumaribo & 0 & 2 & 0,04 \\
\hline \multicolumn{5}{|c|}{ Fisura de paladar y labio leporino } \\
\hline \multirow[t]{2}{*}{ Antioquia } & Dabeiba & 0 & 2 & 0,04 \\
\hline & Granada & 0 & 2 & 0,00 \\
\hline Cauca & Bolivar & 0 & 3 & 0,00 \\
\hline Cesar & San Martin & 0 & 2 & 0,00 \\
\hline Meta & Puerto Gaitán & 0 & 2 & 0,04 \\
\hline Putumayo & Puerto Leguizamo & 0 & 2 & 0,00 \\
\hline
\end{tabular}

Fuente: SIVIGILA - INS, 2015-2018.

Tabla 8. Entidades territoriales con comportamientos inusuales en el grupo de defectos congénitos de etiología cromosómica, Colombia, 2015 - I semestre 2018

\begin{tabular}{|c|c|c|c|c|}
\hline Departamento & Municipio & Media & Observado & Poisson \\
\hline \multicolumn{5}{|l|}{ Síndrome de Down } \\
\hline Antioquia & Envigado & 1 & 4 & 0,02 \\
\hline Bolivar & Clemencia & 0 & 2 & 0,00 \\
\hline \multirow[t]{3}{*}{ Cauca } & Bolivar & 0 & 6 & 0,00 \\
\hline & Patía (El Bordo) & 1 & 3 & 0,03 \\
\hline & Puerto Tejada & 0 & 2 & 0,04 \\
\hline Córdoba & Lorica & 0 & 2 & 0,04 \\
\hline \multirow[t]{3}{*}{ Cundinamarca } & Chía & 0 & 2 & 0,00 \\
\hline & Fusagasugá & 0 & 2 & 0,04 \\
\hline & Zipaquirá & 1 & 4 & 0,02 \\
\hline Guaviare & San Jose del Guaviare & 0 & 2 & 0,00 \\
\hline Magdalena & Ciénaga & 0 & 2 & 0,04 \\
\hline \multirow[t]{2}{*}{ Nariño } & Córdoba & 0 & 3 & 0,00 \\
\hline & Nariño & 0 & 13 & 0,00 \\
\hline Norte de Santander & Sardinata & 1 & 4 & 0,03 \\
\hline \multirow[t]{2}{*}{ Putumayo } & Mocoa & 2 & 8 & 0,00 \\
\hline & Puerto Asís & 0 & 2 & 0,04 \\
\hline Santander & Piedecuesta & 2 & 6 & 0,01 \\
\hline Sucre & Sucre & 1 & 7 & 0,00 \\
\hline \multirow[t]{2}{*}{ Tolima } & Guamo & 0 & 2 & 0,04 \\
\hline & Ibagué & 6 & 11 & 0,03 \\
\hline Valle del Cauca & Cali & 25 & 34 & 0,02 \\
\hline
\end{tabular}

Fuente: SIVIGILA - INS, 2015-2018. 


\section{Identificación de conglomerados espaciales}

Los conglomerados espaciales identifican las relaciones geográficas de los municipios con comportamientos inusuales bajo el criterio de contigüidad, en este sentido, para el caso de los defectos congénitos del sistema nervioso se identifican dos conglomerados. El primero incluye los municipios de Turbo y Apartadó en el departamento de Antioquia, dos municipios contiguos con comportamientos inusuales en casos de anencefalia y microcefalia. El segundo conglomerado incluye a los municipios de San Benito y Sucre en el departamento de Sucre, los dos con comportamientos inusuales en casos de hidrocefalia (Figura 1).

En los casos de defectos congénitos de extremidades se identifican dos conglomerados, el primero en los municipios de Santa Rosa del Sur y San Pablo en el departamento de Bolívar, en donde se identifican comportamientos inusuales en deformidades congénitas de los pies y polidactilia. El segundo conglomerado incluye los municipios de Girón y Bucaramanga en el departamento de Santander, con comportamientos inusuales en defectos por reducción del miembro superior y deformidad congénita de los pies (Figura 2).

Para los defectos congénitos de etiología cromosómica solo se identificaron comportamientos inusuales en casos de síndrome de Down. De esta forma se identificaron dos conglomerados de casos, el primero en el departamento de Cauca e incluye los municipios de Patía y Bolívar, y el segundo se identificó en el departamento de Tolima e incluye los municipios de Guamo e Ibagué (Figura 3).

Finalmente, para el caso de los defectos congénitos relacionados con la fisura de labio y paladar hendido se identifica un solo conglomerado, sin embargo, este involucra dos municipios de dos departamentos diferentes, el municipio de Cumaribo en el departamento de Vichada, con comportamiento inusual en los casos de fisura de labio, y el municipio de Puerto Gaitán en el departamento del Meta, con comportamiento inusual en los casos de fisura de paladar y labio (Figura 4).

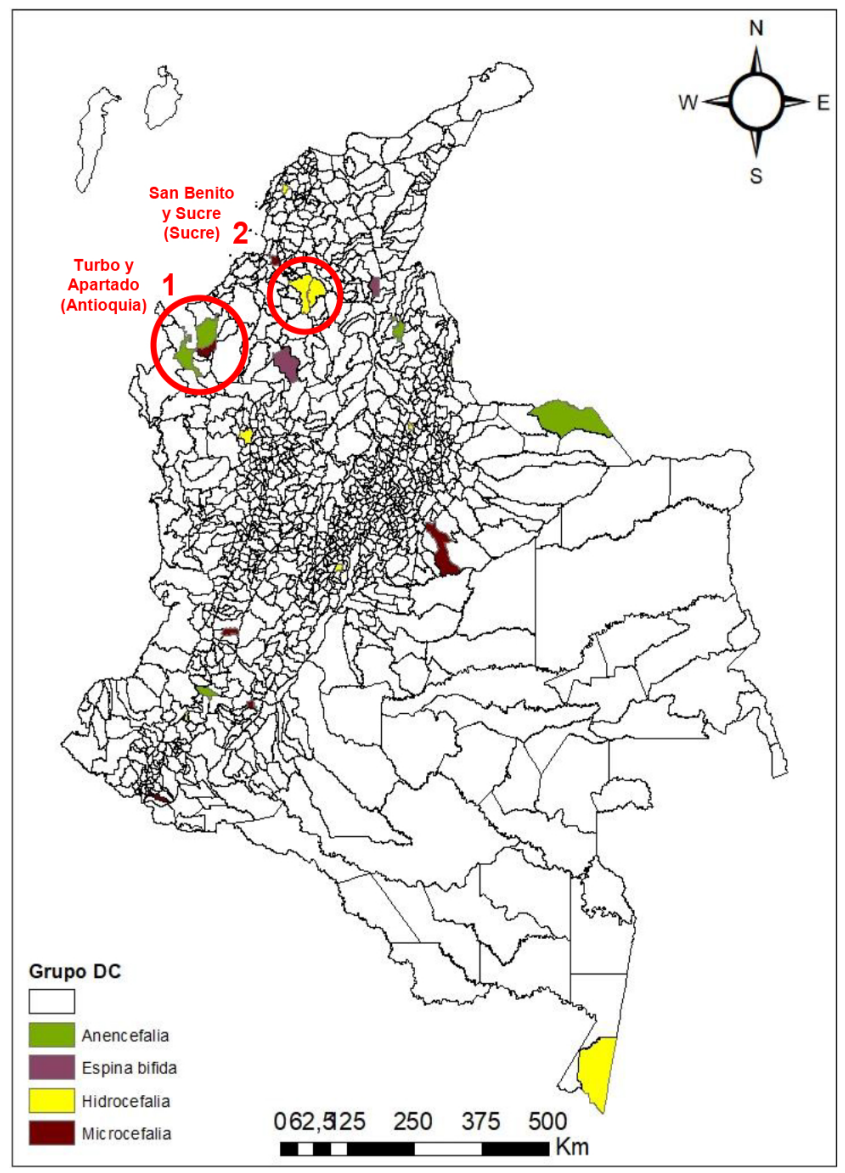

Figura 1. Conglomerados de casos de defectos congénitos del sistema nervioso central, Colombia, I semestre 2018 Fuente: SIVIGILA - INS, 2015-2018. 


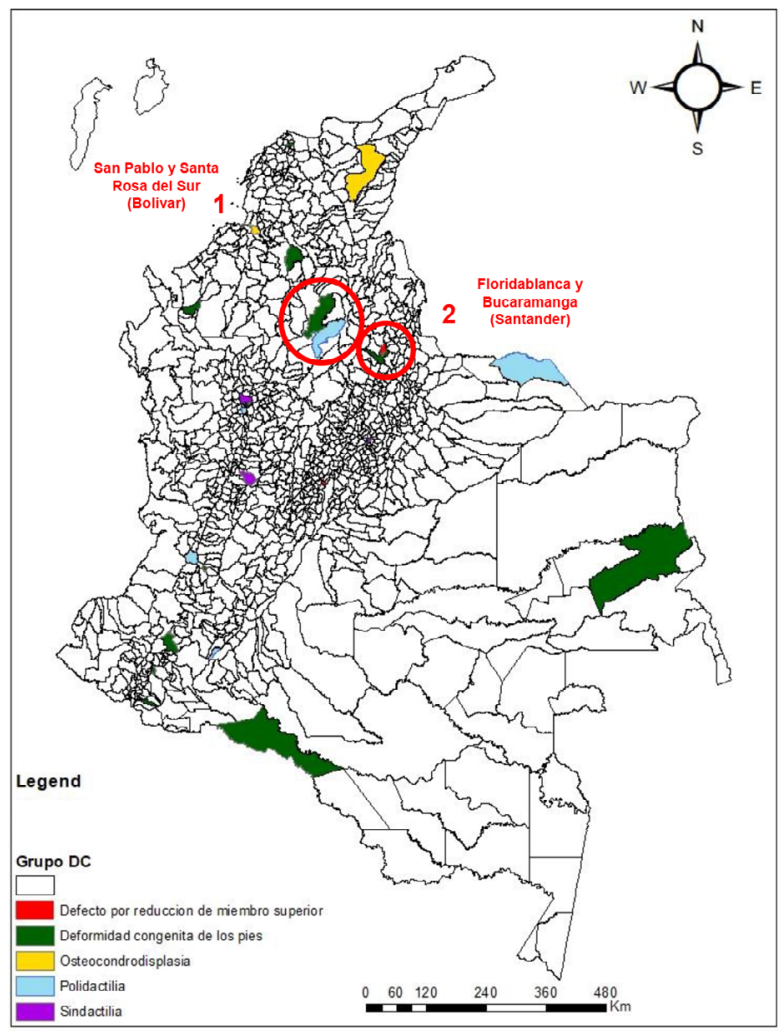

Figura 2. Conglomerados de casos de defectos congénitos de las extremidades, Colombia, I semestre 2018 Fuente: SIVIGILA - INS, 2015-2018.

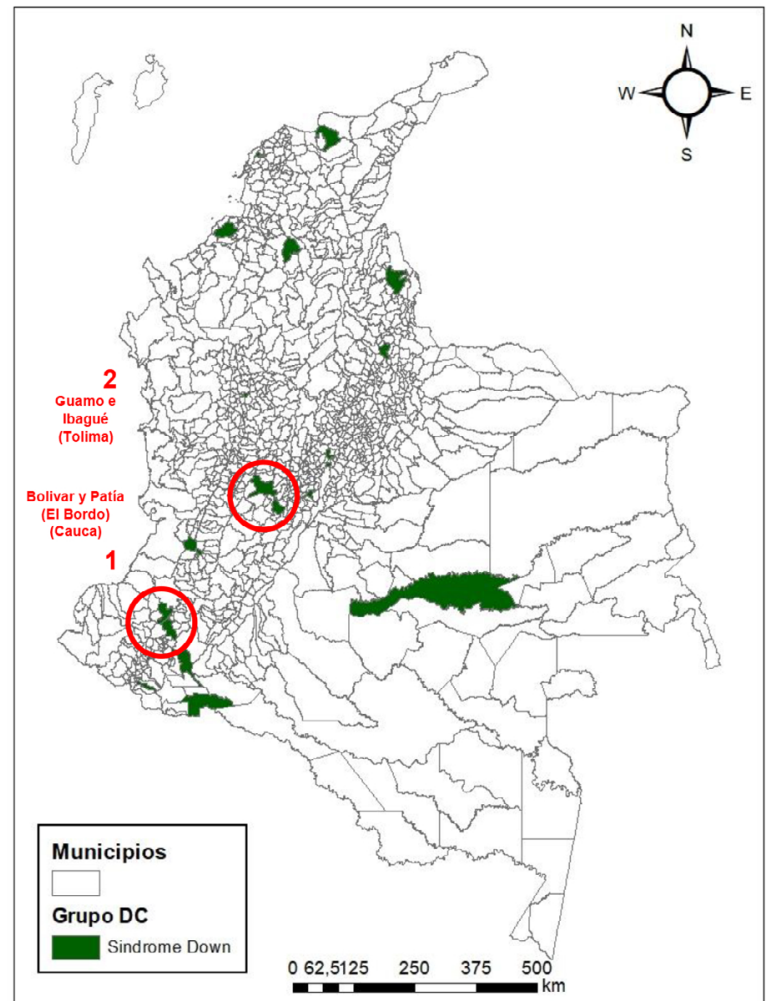

Figura 3. Conglomerados de casos de defectos congénitos cromosómicos, Colombia, I semestre 2018 Fuente: SIVIGILA - INS, 2015-2018. 


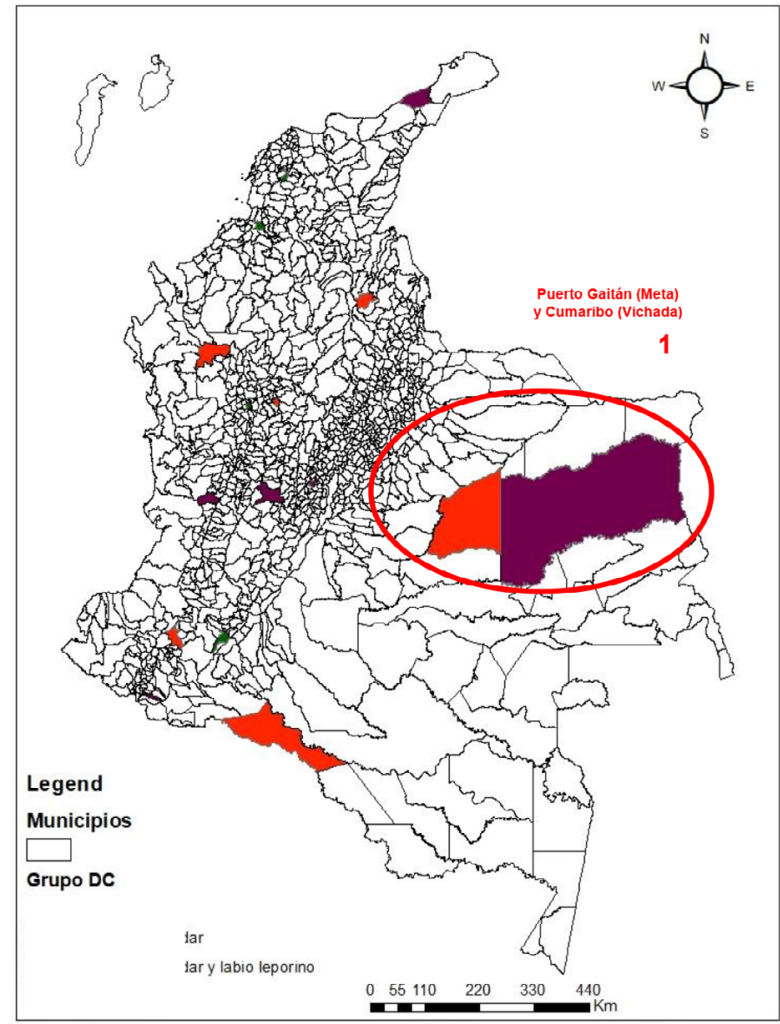

Figura 4. Conglomerado de casos de defectos congénitos de fisura de labio y paladar hendido, Colombia, I semestre 2018

\section{Discusión}

El modelo de monitoreo de comportamientos inusuales o conglomerados de defectos congénitos que se plantea en el presente artículo permite identificar cambios en la prevalencia de los defectos congénitos y detectar patrones inusuales de agregación temporal y/o espacial que puedan sugerir la realización de estudios posteriores para identificar la influencia de factores medioambientales o de exposición individual. En un estudio similar realizado en $\mathrm{Cali}^{12,13}$ en donde se detectaron dos conglomerados de defectos congénitos de baja frecuencia para sirenomelia y ciclopia, generando así el planteamiento de hipótesis y evaluación de factores medioambientales que pudieran estar relacionados con estos defectos congénitos y con los lugares donde se identificaron los casos.

Un estudio publicado en 2002 por el Departamento de Medio Ambiente y Salud del Estado de Colorado (Estados Unidos) estimó que entre el 7\% y el 10\% de todos los defectos congénitos en esa área se explicaban por factores ambientales; mientras que la herencia multifactorial (combinación entre factores ambientales y genéticos) explicó del 20 al $25 \%$, y causas desconocidas explicadas entre 50 y $60 \%{ }^{2,14}$. Sin embargo, en países con bajos recursos, es posible que el peso de los factores ambientales asociados al lugar de residencia en los microentornos contaminados sea mayor que el referido en los países más ricos ${ }^{13}$.

Los comportamientos inusuales de los defectos congénitos de sistema nervioso central se concentran en anencefalia, espina bífida, hidrocefalia y microcefalia. Lo observado coincide con otros países que en 2016 reportaron un incremento inusual de estos casos debido al brote de Zika, donde se confirmó la relación de la infección por este agente y la ocurrencia de microcefalia. Debido a la alerta emitida por la Organización Mundial de la Salud, diseñaron el Plan de Respuesta Estratégica, donde se contempló el fortalecimiento de los sistemas de vigilancia para monitorizar esta infección y sus complicaciones $^{15}$. Colombia fue uno de los países que intensificó la vigilancia de las microcefalias y otros defectos congénitos del sistema nervioso central desde el $2016^{16}$, lo que requirió el diseño del protocolo específico y la generación de circulares externas a todos los territorios para fortalecer la detección y notificación de defectos congénitos de sistema nervioso central, lo que podría explicar que el incremento de estos casos.

El comportamiento de los defectos congénitos relacionados con el tubo neural descrito en este 
documento coincide con otro estudio desarrollado en Argentina, donde este grupo de defectos presentó una prevalencia de 8,8 casos por 10000 nacidos vivos ${ }^{17}$. Los principales defectos descritos en este grupo fueron la anencefalia (1,8 casos), encefalocele (1,2 casos), espina bífida (5,7 casos), hidrocefalia (6,9 casos), holoprosencefalia (2,1 casos) y microcefalia ( 2,5 casos). Cifras similares para Colombia, excepto encefalocele y holoprosencefalia que tiene un comportamiento mayor de lo reportado en dicho estudio ${ }^{17}$.

Un estudio realizado sobre la prevalencia de defectos congénitos en Risaralda muestra que el sistema más afectado fue el musculoesquelético, con una tasa de 26,4 por 10000 nacidos vivos. La tasa de alteraciones en los miembros fue de 13,4 por 10000 nacidos vivos. Se ha descrito que este tipo de defectos está relacionado con una falla en el desarrollo durante el periodo de gestación, en otros casos se han identificado problemas con las bandas amnióticas o disrupción vascular, que producen reducción de los miembros ${ }^{18}$.

En cuanto a la polidactilia, en el estudio actual la prevalencia fue de 7,54 por 10000 nacidos vivos en 2017 , cifra que se encuentra por encima de lo reportado en el estudio de Risaralda (4,24 por 10000 nacidos vivos) y por encima de lo reportado por la ECLAMC $(3,14)$, datos que respaldan y son concordantes con la identificación del comportamiento inusual en el país para este tipo de defecto.

En un estudio realizado en el Hospital Universitario del Valle entre julio de 2011 y junio de 2012 se identificaron las prevalencias más altas en el grupo de defectos de las extremidades con 79,37 casos por cada 10000 nacidos vivos, específicamente en polidactilia 24,69 , sindactilia 8,81 , e hipoplasia/defectos longitudinales de las extremidades superiores 5,2912. Al realizar la comparación con los resultados de la identificación de comportamientos inusuales, en especial la cifra para polidactilia es concordante con lo observado en el departamento del Valle, en donde se presentó el mayor número de casos para este tipo de defecto.

Barten sugiere que, en la actualidad, los rápidos procesos de urbanización en las ciudades generan concentraciones de pobreza y las inequidades en la salud afectan a las comunidades marginadas ${ }^{19}$, aspecto para tener en cuenta especialmente en las poblaciones desplazadas. De igual forma, teniendo en cuenta el índice de pobreza por departamento de Colombia, es necesario indagar sobre las condiciones de vida de las poblaciones del Cauca, Huila, Sucre, Nariño y Bolívar las cuales se encuentran dentro de las 10 primeras con mayores índices de pobreza según el DANE, y que se observan en este estudio con resultados inusuales en el comportamiento de los defectos congénitos de las extremidades.

En el estudio realizado en Cali entre 2011 y 2012 sobre los defectos congénitos en un hospital de tercer nivel se observó en $11^{\circ}$ lugar de frecuencia las gastrosquisis ( 8,81 por cada 10000 nacidos vivos) y en el $26^{\circ}$ lugar de frecuencia los onfaloceles (3,52 por cada 10000 nacidos vivos) ${ }^{12}$. Cifras que son elevadas comparadas con las calculadas para Colombia en 2017 para gastrosquisis (3,40 por cada 10000 nacidos vivos) y para onfalocele ( 0,90 por cada 10000 nacidos vivos). Sin embargo, para la gastrosquisis se observa que a partir de 2015 se presenta un aumento en la prevalencia cada año, lo que se podría justificar por la presencia de comportamientos inusuales en Arauca y Nariño. Con respecto al comportamiento del onfalocele la cifra reportada es menor a la descrita por el estudio de comparación, para el primer semestre de 2018 sí se observa un aumento en la prevalencia comparado con los tres años anteriores, lo cual podría justificar la presencia de comportamientos inusuales en Acacias - Meta.

El patrón geográfico identificado en los conglomerados de defectos congénitos de extremidades no permite inferir características comunes, ya que circunscribe los municipios de Santa Rosa del Sur y San Pablo en el Departamento de Bolívar, en los que está presente el denominado sector primario, con la minería como actividad fundamental, seguida de la agricultura y la ganadería, características que no comparten los municipios de Girón y Bucaramanga, en donde se identificó el segundo conglomerado y que se orientan a los sectores de comercio, servicios, industria y turismo. Por lo anterior, son necesarios estudios analíticos posteriores que permitan identificar factores ambientales, sociodemográficos, culturales, y otros, que expliquen la variabilidad geográfica encontrada en este tipo de defectos.

Así mismo, se requiere profundizar en el análisis de lo que aporta la literatura como posibles factores etiológicos para los defectos de las extremidades y correlacionarlo con los hallazgos particulares de cada municipio. Se conoce que la polidactilia es heterogénea, se ha encontrado asociada con ancestros afrodescendientes en mayor frecuencia que en otros grupos poblacionales, con consanguinidad de los padres y en menor proporción por acción de un gen recesivo ligado a $\mathrm{X}^{20}$. En diferentes estudios se ha 
observado una asociación con los antecedentes de otra malformación en la familia, lo que permite vincular los factores hereditarios con la etiología ${ }^{21}$; e igualmente se ha identificado que la diabetes materna o gestacional es un factor de riesgo de esta anomalía preaxialmente en el $\mathrm{pie}^{22}$. En cuanto a las deformidades de los pies, han sido asociadas con la exposición a rayos $\mathrm{X}$ no abdominales ${ }^{20}$.

Frente a los defectos congénitos del sistema circulatorio, en el estudio realizado en Risaralda entre 2010 y $2013^{18}$ el primer lugar de defectos congénitos mayores lo ocuparon las cardiopatías congénitas con una prevalencia de 8,72 por 10000 nacidos vivos. En otros estudios este tipo de defectos no ocupa el primer lugar en prevalencia, sin embargo, sus cifras continúan siendo elevadas comparadas con el presente estudio. En el estudio realizado en Cali ${ }^{12}$ las cardiopatías se ubicaron en el quinto lugar con una prevalencia de 14,11 por 10000 nacidos vivos y en el estudio realizado en tres ciudades colombianas entre 2001 y $2008^{5}$ las cardiopatías congénitas se ubicaron en el séptimo lugar, con una prevalencia de 15,7 por 10000 nacidos vivos. Pese a lo anterior, las cifras comparadas en los últimos tres años y el primer semestre de 2018 han tenido un aumento significativo, lo cual podría explicar la identificación de los comportamientos inusuales en Antioquia, Boyacá, Santander y Risaralda.

Para los comportamientos inusuales de los defectos de fisura de paladar, fisura de labio y fisura del paladar con fisura de labio, lo reportado por la literatura es que estos defectos congénitos son de etiología multifactorial, entre los que se han descrito: factores hereditarios, factores genéticos, ambientales, nutricionales, tabaquismo, edad materna menor a 20 años o mayor a 35 años, embarazo múltiple, exposición a fertilizantes y plaguicidas y otros factores (mecánicos, radiaciones, químicos, biológicos del grupo STORCH) ${ }^{23,24}$. Su aumento en el sistema de vigilancia de Colombia puede estar atribuido a la mejora en el acceso a los servicios de salud para el diagnóstico temprano y su respectiva notificación, teniendo en cuenta que en la última Encuesta de Demografía y Salud el porcentaje de afiliación al Sistema de Seguridad Social en Salud fue del 94,1\%. Así como también este comportamiento puede atribuirse a que en los últimos cinco años hay un leve aumento en la atención de parto institucional, con un $96,9 \%$ para el $2015^{25}$.

Los defectos congénitos relacionados con fisuras orales descritos anteriormente tienen un comportamiento similar a lo observado en otro estudio desarrollado en Argentina, donde se estimó una prevalencia de 16,4 casos por 10000 nacidos $\operatorname{vivos}^{17}$. Los principales defectos de este grupo descritos fueron el paladar hendido (3,2 casos por 10000 nacidos vivos), fisura labial (1,9 casos por 10000 nacidos vivos) y fisura labio palatina (11,1 casos por 10000 nacidos vivos). Cifras similares para Colombia, excepto fisura de labio (2,06 casos por 10000 nacidos vivos) que tiene un comportamiento mayor de lo reportado en dicho estudio $^{17}$.

Al realizar el análisis por conglomerados espaciales para estos defectos que reportaron comportamientos inusuales se identifica que hay un comportamiento particular, ya que es el único que involucra dos municipios de dos departamentos diferentes, el municipio de Cumaribo en el departamento de Vichada, con comportamiento inusual en los casos de fisura de labio, y el municipio de Puerto Gaitán en el departamento del Meta, con comportamiento inusual en los casos de fisura de paladar y labio. Con relación a los descrito anteriormente, ambos municipios pueden tener similitudes en cuanto a múltiples factores que explican la presencia de estos conglomerados, tales como consanguinidad parental, que se observa de forma frecuente en los grupos indígenas ${ }^{26}$; embarazos en adolescentes, donde se reportan las tasas de fecundidad más altas en las menores de 19 años ${ }^{27}$; factores biológicos del grupo STORCH (sífilis, toxoplasma, rubeola, citomegalovirus y herpes) junto con la exposición a fertilizantes y plaguicidas utilizados en los cultivos agroindustriales e ilícitos ${ }^{28,29}$.

Los comportamientos inusuales en el grupo de defectos congénitos de etiología cromosómica solo se identificaron para los casos con diagnóstico de síndrome de Down, esta es la cromosomopatía más frecuente, causada por la no disyunción del cromosoma 21 y con factores de riesgo multifactoriales en donde influye la interacción de factores genéticos y ambientales. Dentro de los principales factores de riesgo se ha identificado la edad materna avanzada $^{30}$. Adicionalmente, la consanguinidad se considera un factor de riesgo que aumenta la posibilidad de una condición genética recesiva independiente de la edad materna ${ }^{31}$.

Se han identificado en total 21 municipios con comportamiento inusuales en la notificación de casos de síndrome de Down, lo cual puede verse afectado por la diferente distribución de las edades maternas, pero también por el diagnóstico prenatal y el acceso a los servicios de salud, que de alguna manera son los responsables de la notificación e identificación de los casos al sistema de vigilancia. En algunos estudios realizados en Cali, municipio con comportamiento 
inusual y con el mayor número de casos esperados y observados, se ha identificado la ocurrencia de alteraciones cromosómicas en recién nacidos, principalmente en hijos de mujeres mayores de 30 años ${ }^{19}$. Igual situación fue identificada en un estudio realizado en población del departamento del Norte de Santander en $2004^{32}$.

La reciente evidencia ${ }^{31}$ en torno al tema del síndrome de Down ha identificado una posible relación entre la consanguinidad y la ocurrencia de este tipo de defecto cromosómico. En este sentido, una investigación realizada en municipios al norte del departamento del Cauca $^{33}$ identificó de forma indirecta una alta frecuencia de consanguinidad próxima y remota, lo cual podría estar relacionado con la aparición de conglomerado de defectos cromosómicos en esta zona.

Al realizar el análisis por conglomerados espaciales para los defectos congénitos de sistema nervioso central que reportaron comportamientos inusuales se identifica a Turbo y Apartadó en el departamento de Antioquia, dos municipios contiguos con comportamientos inusuales en casos de anencefalia y microcefalia. La anencefalia puede estar explicada por la condición nutricional de la población residente en esta zona del país, teniendo en cuenta que se ha reportado que cerca del $50 \%$ de menores de 14 años viven en desnutrición crónica ${ }^{34}$; se afirma que cerca del $87,9 \%$ de la población se encuentra en niveles de pobreza y el $80,8 \%$ en miseria ${ }^{35}$, lo que podría suponer que la mayoría de la población en edad fértil convive con esta situación nutricional.

Turbo y Apartadó son municipios que hacen parte de la zona del Urabá Antioqueño, donde su principal actividad económica y productiva es el cultivo y exportación del banano, por lo que hacen uso intensivo de plaguicidas y agroquímicos para el control de plagas y enfermedades del cultivo del banano, que no solo explican la exposición frecuente de la población a estos agentes tóxicos, sino también la contaminación del agua y la alta concentración en la atmósfera ${ }^{35}$. Se ha descrito que la exposición a plaguicidas y la presencia de antecedentes familiares de malformación congénita tiene asociación estadísticamente significativa para la ocurrencia de estos defectos congénitos ${ }^{36}$.

El segundo conglomerado de defectos congénitos de sistema nervioso central incluye a los municipios de San Benito y Sucre en el departamento de Sucre, los dos con comportamientos inusuales en casos de hidrocefalia. Se debe tener en cuenta que la principal actividad económica de estos municipios es la agricultura, con la existencia de venta, distribución, uso y comercialización de plaguicidas. Dentro del mapa de riesgos de plaguicidas del departamento, estos dos municipios están categorizados en uso de plaguicidas moderadamente peligrosos (OMS) y altamente tóxicos (Decreto 1843 de 1991) ${ }^{37}$. Se ha descrito que la hidrocefalia está relacionada con la exposición a fertilizantes y plaguicidas ${ }^{36}$, así como también el retraso del crecimiento fetal, antecedentes de malformaciones congénitas en la familia, factores físicos y enfermedades agudas de la madre en el primer trimestre del embarazo ${ }^{36}$.

La salud pública está influenciada por factores ambientales y por factores relacionados con la habitabilidad de los lugares, por lo anterior, no es sufiente un análisis decsriptivo sobre el comportamiento de un evento de interés en salud pública con sus características poblacionales relevantes, sino que tambien implica la georreferenciación con las ubicaciones especificas de estos eventos y su posible relación con los servicios disponibles, el potencial riesgo ambiental y las condiciones de vulnerabilidad que podrían afectar a las comunidades.

Las condiciones particulares de cada municipio y/o departamento y el comportamiento de los defectos congénitos en algunas áreas podría indicar que las mujeres en estado de gestación son susceptibles a mayores riesgos en zonas particulares, y que este riesgo en particular podría ser el resultado de diversas inequidades en salud generadas por las interacciones sociales, ambientales y comportamentales que favorecen la exposición de las comunidades a diversos agentes los cuales inciden directamente sobre la salud.

Es necesario contar con más estudios para evaluar los factores ambientales, exposición a contaminantes, factores sociodemográficos, factores culturales, inequidad y desigualdad social, y la susceptibilidad de la población en las zonas que se identificaron en el presente estudio con comportamientos inusuales de defectos congénitos, con el fin de enriquecer los análisis posteriores y proporcionar una evidencia significativa que oriente la toma de decisiones en salud pública para todos los actores involucrados.

\section{Recomendaciones}

Es importante mencionar que, para los defectos congénitos del sistema nervioso central, se deben tener en cuenta factores genéticos, infecciosos o ambientales presentes en los territorios, lo cual podría impactar en la disminución o control de dichos trastornos congénitos. 
Adicionalmente, para los defectos congénitos del tubo neural (espina bífida y anencefalia) se requieren acciones preventivas de nivel primario, como la suplementación con ácido fólico desde el periodo preconcepcional y la importancia de la asistencia a controles prenatales continuos y de calidad para la identificación de posibles factores de riesgo: edad de la madre, exposición a sustancias teratogénicas, desnutrición y causas genéticas. Por ejemplo, en América Latina varios países han implementado políticas para la fortificación de harina de trigo y otros cereales ${ }^{38}$. Para los defectos congénitos del sistema circulatorio se ha identificado el nacimiento prematuro, embarazo múltiple y edad materna avanzada, este último factor también está relacionado con el síndrome de Down, los anteriores son elementos que podrían estar relacionados con la incidencia y prevalencia, por lo que es necesario fortalecer la identificación temprana de estos factores de riesgo en las mujeres en edad fértil y tenerlos en cuenta para orientar las consultas preconcepcionales como estrategias de prevención ${ }^{39}$.

\section{Agradecimientos}

Al Instituto Nacional de Salud por la posibilidad de diseñar nuevas metodologías de análisis de la información. A los profesionales de las instituciones de salud, a los referentes de vigilancia en salud pública de las Secretarías Departamentales, Distritales y Municipales de Salud por desarrollar el proceso de vigilancia de los congénitos en su territorio para la generación de información que oriente la toma de decisiones en salud pública.

\section{Conflicto de interés}

El autor de este artículo no declara conflicto de interés. La fuente de financiación de este provino de recursos propios.

\section{Referencias}

1. Fonseca R. Malformaciones congénitas: Nuevos desafíos para la Salud Pública. Pediatria. (Asunción). 2018; 45 (1): 05-07. doi: https://doi.org/10.31698/ ped. 45012018001

2. Christianson A, Howson CP, Modell B. Global report on birth defects: The hidden toll of dying and disabled children. March of dimes birth defects foundation: White Plains (NY): March of Dimes Birth Defects Foundation, 2006.

3. Sitkin NA, Ozgediz D, Donkor P, Farmer DL. Congenital anomalies in low- and middle-income countries: The unborn child of global surgery. World J Surg. 2015; 39(1): 36-40. doi: https://doi. org/10.1007/s00268-014-2714-9

4. Organización Panamericana de la Salud (OPS). Boletín Informativo: Malformaciones Congénitas. Managua: OPS; Junio de 2015. 6 p.

5. Zarante I, Franco L, López C, Fernández N. Frecuencia de malformaciones congénitas: evaluación y pronóstico de 52.744 nacimientos en tres ciudades colombianas. Biomédica. 2010; 30(1): 65-71. doi: https://doi.org/10.7705/biomedica. v30i1.154

6. Avila-Mellizo GA. Informe de evento defectos congénitos, Colombia, 2017. Bogotá: Instituto Nacional de Salud; 20 de abril de 2018. Informe de evento núm: FOR-R02.4000-001.

7. Organización Mundial de la Salud. 63. ${ }^{a}$ Asamblea Mundial de la Salud: WHA63.17. Ginebra (Suiza): Organización Mundial de la Salud. 2010. http:// apps.who.int/gb/ebwha/pdf_files/WHA63/A63 R17-sp.pdf?ua=1\&ua=1\&ua=1

8. German RR, Lee LM, Horan JM, Milstein RL, Pertowski CA, Waller MN; Guidelines Working Group Centers for Disease Control and Prevention (CDC). Updated guidelines for evaluating public health surveillance systems: recommendations from the Guidelines Working Group. MMWR Recomm Rep. 2001;50(RR-13):1-35; quiz CE1-7.

9. Martínez N. De la información a la acción: la vigilancia de la salud pública. Rev Esp Salud Publica. 2000; 74(1): 1-5.

10. Marie GC, Soria JB, Moliner RB, Cardenas AZ, Cañizares PF. Métodos para la vigilancia de eventos en salud. Rev Cubana Hig Epidemiol. 2000; 38(3): 157-166.

11. Estudio Colaborativo Latinoamericano de Anomalías Congénitas (ECLAMC). Manual operacional. Octava edición. Río de Janeiro: ECLAMC, 2009.

12. Ramírez-Cheyne J, Pachajoa H, Ariza Y, Isaza C, Saldarriaga W. Defectos congénitos en un hospital de tercer nivel en Cali, Colombia. Rev Chil Obstet Ginecol. 2015; 80(6): 442-449. doi: http://dx.doi. org/10.4067/S0717-75262015000600003

13. Cuartas D, Ariza Y, Pachajoa H, Mendez F. Analysis of the spatial and temporal distribution of birth defects between 2004-2008 at a third-level hospital in Cali, Colombia. Rev Colombia Medica. 2011; 42(1): 9-16. doi: https://doi.org/10.25100/ cm.v42i1.745

14. Colorado Disease Control and Environmental Epidemiology Division. Analysis of Birth Defect Data in the Vicinity of the Redfield Plume Area 
in Southeast Denver County, 1989-1999. Denver: Colorado Department of Public Health and Environment, Disease Control \& Environmental Epidemiology Division, 2002.

15. World Health Organization. Zika Strategic Response Plan. Ginebra (Suiza): WHO 2016: 1-50.

16. Instituto Nacional de Salud. Protocolo de vigilancia intensificada de microcefalia y otros defectos congénitos del sistema nervioso central por virus zika. Bogotá (Colombia). INS 2016; 1-20.

17. Groisman B, Bidondo M, Duarte S, Tardivo A, Barbero P, Liascovich R. Epidemiología descriptiva de las anomalías congénitas estructurales mayores en Argentina. MEDICINA (Buenos Aires) 2018; 78: 252-257.

18. Porras-Hurtado G, León-Castañeda O, MolanoHurtado J, Quiceno S, Pachajoa H, Montoya J. Prevalencia de defectos congénitos en Risaralda, 2010-2013. Biomédica 2016; 36 (4): 556-563. doi: https://doi.org/10.7705/biomedica.v36i4.2771

19. Barten F, Flores W, Hardoy A. La inequidad en salud: Hacia un abordaje integral. Buenos Aires: IIED-América Latina Publicaciones, 2008.

20. Lopez-Camelo JS, Orioli IM. Heterogeneous rates for birth defects in Latin America: hints of causality. Genet Epidemiol. 1996. 13(5): 469-481. https://doi.org/10.1002/(SICI)10982272(1996)13:5<469::AID-GEPI3>3.0.CO;2-Y

21. Perez-Molina JJ, Alfaro-Alfaro N, López-Zermeno M, García-Calderón M. Polydactyly in 26.670 consecutive births. The clinical characteristics, prevalence and risk factors. Bol Med Hosp Infant Mex.1993; 50(11): 803-808.

22. Martínez-Frías ML, Bermejo E, Cereijo A. Preaxial polydactyly of feet in infants of diabetic mothers: epidemiological test of a clinical hypothesis. Am J Med Genet; 1992.42(5): 643-646. doi: https://doi. org/10.1002/ajmg.1320420502

23. Caraguay Medina NA. Prevalencia y predisposición genética de labio leporino y paladar hendido en pacientes pediátricos. Estudio a realizar en el Hospital Francisco Icaza Bustamante durante el período de enero del 2015 a enero del 2017. Guayaquil: Universidad de Guayaquil; 2018.

24. Balbis Cabrera M, Cabrera Cuellar . La fisura labiopalatina no es inevitable. Medisur 2018; 16(1): 1-3.

25. Ministerio de Salud y Protección Social. Encuesta Nacional de Demografía y Salud: Tomo 1 y 2. Bogotá: MSPS, 2015.

26. Lardoeyt R, Rodriguez R, Jijón M. Consanguinidad y agregación familiar en personas con discapacidad intelectual en la República del Ecuador. Rev Cubana
Genet Comunit. 2011; 5(2-3): 64-69.

27. Observatorio del bienestar de la niñez. Embarazo en adolescentes: generalidades y percepciones. Bogotá: Instituto Colombiano de Bienestar Familiar (ICBF): 2015: 1-36.

28. Oficina de las Naciones Unidas contra la Droga y el Delito. Atlas de la caracterización Regional de la problemática asociada a las drogas ilícitas en el departamento de Vichada. Bogotá (Colombia): UNODC-Minjusticia, 2013: 1-101.

29. Alcaldia municipal. Plan de Desarrollo Municipal "Voluntad para el Progreso", municipio de Puerto Gaitán, Meta, 2016-2019. Puerto Gaitán (Meta): 2016; 1-171.

30. Grupo de trabajo de la Sociedad Española de Ginecología y Obstétrica (SEGO). Guía de práctica clínica: Diagnóstico prenatal de los defectos congénitos. Cribado de anomalías cromosómicas. Diagnóstico Prenatal. 2013; 24 (2): 57-72. doi: https://doi.org/10.1016/j.diapre.2012.06.013

31. Ray A, Oliver TR, Halder P, Pal U, Sarkar S, Dutta, et al. Risk of down syndrome birth: Consanguineous marriage is associated with maternal meiosis-II nondisjunction at younger age and without any detectable recombination error. Am J Med Genet A. 2018; 176(11): 2342-2349. doi: https://doi. org/10.1002/ajmg.a.40511

32. Palomino J, Pontón M, Canedo Y, Osorio A, Callejas A, Molina M. Edad materna y/o paterna como factor de riesgo genético en niños del centro de atención materno infantil de la universidad de pamplona (caimiup) y el instituto la aurora de la ciudad de pamplona, Norte de Santander, Colombia. Rev BISTUA. 2004; 2(1): 77-90. doi: https://doi. org.10.24054/01204211.v1.n1.2004.16

33. Giraldo A, Pino W, Pineda M, García F, Freyle D, Nova F, et al. Los niños del Cauca: descripción de un foco de raquitismo dependiente de la vitamina $\mathrm{D}$ tipo II. Biomédica. 1993; 13(3): 3-13. doi: https:// doi.org/10.7705/biomedica.v13i3.2059

34. Carmona-Fonseca J. Alimentación y estado nutricional de los niños en zonas palúdicas de Antioquia (Colombia). MedUNAB 2011; 14(2): $94-$ 102.

35. Gobernación de Antioquia. Lineamientos de política sobre uso y manejo mesurado de plaguicidas con énfasis en el sector agropecuario y forestal del departamento Medellín: Corantioquia, 2005; 1-140.

36. Concepción L, Benítez S. Factores de riesgo prenatales y su asociación a malformaciones congénitas en un hospital universitario de referencia. Pediatria. 2018; 45(1): 8-16. doi: https:// doi.org/10.31698/ped.45012018002 
37. Gobernación de Sucre. Análisis de la situación de salud del Departamento de Sucre. 2011. 2012: $1-230$.

38. Sierra M, Rumbo J, Salazar A, Sarmiento K, Suarez F, Zarante I. Perinatal mortality associated with congenital defects of the central nervous system in Colombia, 2005-2014. J Community Genet. 2019; 10(4): 515-521. doi: https://doi.org/10.1007/ s12687-019-00414-x

39. Reller M, Strickland M, Riehle-Colarusso T, Mahle W, Correa A. Prevalence of congenital heart defects in metropolitan Atlanta, 1998-2005. J Pediatr. 2008; 153(6): 807-813. doi: https://doi.org/10.1016/j. jpeds.2008.05.059 